\section{Hausärzte sollten Kürzungen bei Psychoziffern nicht hinnehmen}

_ Wir erinnern uns: Im Jahr 2009 wurden die Leistungen nach den Nrn. 35100 und 35110 EBM mit Fallwertzuschlägen von 3 Euro gefördert. Diese Regelung wurde 2010 zwar wieder aufgehoben, die Einstufung psychosomatischer Leistungen als besonders förderungswürdig besteht aber fort - und dieser Umstand wird nun wieder interessant.

Mit dem GKV-Versorgungsstrukturgesetz wurde das Vergütungssystem regionalisiert und flexibilisiert. Die KVen

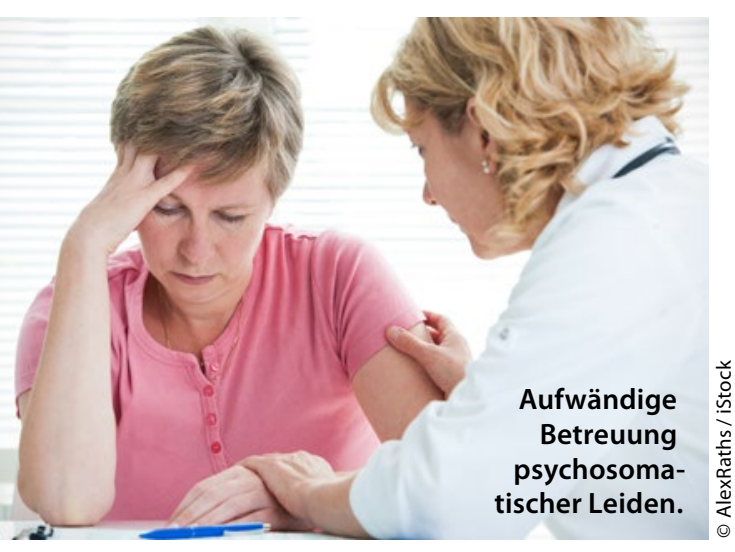

erhielten die Möglichkeit, auf regionaler Ebene mit den Krankenkassen Preiszuschläge für förderungswürdige Leistungen festzulegen. Auch besonders förderungswürdige Leistungserbringer können definiert werden, insbesondere in strukturschwachen Gebieten. Dazu haben Kassen und KBV auf Bundesebene Kriterien festgelegt.

\section{MMW-KOMMENTAR}

Die Versorgung psychogener Erkrankungen obliegt in Deutschland überwiegend den Hausärzten. In den Erhebungen zur Gesamtprävalenz psychischer Erkrankungen in hausärztlichen Praxen schwanken die ermittelten Raten zwischen 21\% und 52\% (Üstün) Sartorius 1995). Andere Quellen beschreiben eine noch höhere Schwankungsbreite zwischen $17,5 \%$ und $64,3 \%$.

Nach Angaben des Fehlzeiten-Reports 2012 des Wissenschaftlichen Instituts der AOK (WIdO) ist die Zahl der Arbeitsunfähigkeitsfälle wegen psychischer Störungen seit 1994 um $120 \%$ gestiegen. Trotz der im internationalen Vergleich relativ gut ausgebauten psy-

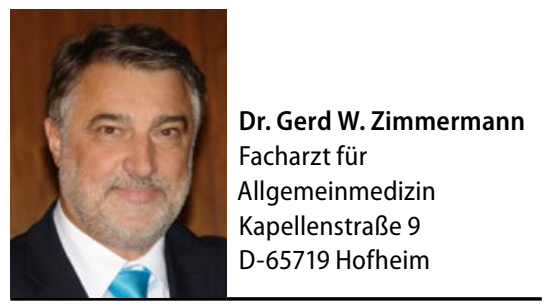

chotherapeutischen Versorgung in Deutschland liegt der Anteil derjenigen, die bei einer psychischen Störung irgendwann einmal eine angemessene professionelle Intervention erhalten, in Deutschland nur bei knapp 40\% (Schulz et al. 2008). Die meisten Menschen mit psychischen Störungen werden immer noch zu selten, zu spät und zu wenig leitliniengerecht behandelt.

Bedauerlicher Alltag angesichts solcher Zahlen ist, dass hausärztliche Praxen, die sich dieser volkswirtschaftlichen Herausforderung stellen und psychosomatisch erkrankte Patienten adäquat und leitliniengerecht behandeln, wegen der beschriebenen Schwankungsbreite innerhalb der Fachgruppe statistisch auffällig werden. Die Folge ist oft eine pauschale Kürzung des erzielten Honorars auf der Grundlage des "offensichtlichen Missverhältnisses zur Vergleichsgruppe“.

In solchen Fällen sollte man deshalb die Forderung nach einer (zumindest repräsentativen) Einzelfallprüfung bei der Beurteilung der Wirtschaftlichkeit der Behandlung stellen und ggf. konsequent bis in die juristische Ebene verfolgen.

\title{
Abrechnung der Behandlung zur "Unzeit"
}

_ Die Nr. 01100 EBM kann berechnet werden, wenn ein Patient den Vertragsarzt unvorhergesehen zwischen 19 und 22 Uhr an einem Werktag bzw. zwischen 7 und 19 Uhr an einem Ruhetag (Samstag, Sonntag, gesetzlicher Feiertag, 24. und 31. Dezember) in Anspruch nimmt. Findet der Kontakt zwischen 22 und 7 Uhr bzw. zwischen 19 und 7 Uhr an einem Ruhetag statt, wird die Nr. 01101 angesetzt. Die Leistungen sind mit 20,45 bzw. 32,66 Euro bewertet und gehen nicht in die Plausibilitätsprüfung nach Zeitvorgaben ein.

\section{MMW-KOMMENTAR}

Die Nrn. 01100/01101 können nicht im Zusammenhang mit Hausbesuchen berechnet werden. Möglich ist dies allerdings bei der Nr. 01102 für die Inanspruchnahme des Vertragsarztes an Samstagen zwischen 7 und 14 Uhr, und zwar wenn Besuche in be- schützenden Wohnheimen bzw. Pflege-oder Altenheimen mit Pflegepersonal angefordert werden, die nach Nr. 01413 berechnungsfähig sind. In diesem Fall ist die Unzeitziffer möglich. Sie wird mit 10,54 Euro vergütet. Auch bei der Nr. 01102 gibt es keine Zeitvorgabe. Alle genannten Leistungen können zu den angegebenen Zeiten außerdem berechnet werden, wenn es am Ende lediglich zu einer telefonischen Kontaktaufnahme mit dem Patienten kommt. 